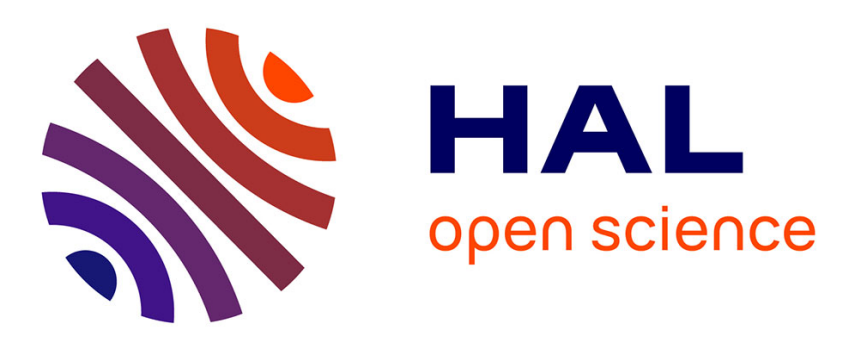

\title{
A 2-d classification of diseases based on age-specific death rates
}

\author{
Peter Richmond, Bertrand Roehner
}

\section{To cite this version:}

Peter Richmond, Bertrand Roehner. A 2-d classification of diseases based on age-specific death rates. Physica A: Statistical Mechanics and its Applications, 2018, 492, pp.2281-2291. 10.1016/j.physa.2017.11.142 . hal-01723098

\section{HAL Id: hal-01723098 \\ https://hal.sorbonne-universite.fr/hal-01723098}

Submitted on 5 Mar 2018

HAL is a multi-disciplinary open access archive for the deposit and dissemination of scientific research documents, whether they are published or not. The documents may come from teaching and research institutions in France or abroad, or from public or private research centers.
L'archive ouverte pluridisciplinaire HAL, est destinée au dépôt et à la diffusion de documents scientifiques de niveau recherche, publiés ou non, émanant des établissements d'enseignement et de recherche français ou étrangers, des laboratoires publics ou privés. 


\section{A 2-d classification of diseases based on age-specific death rates}

\section{Peter Richmond ${ }^{1}$ and Bertrand M. Roehner ${ }^{2}$}

Abstract Age specific mortality curves exhibit an age $t_{c}$ (about 10 years) which plays a crucial role in that the mortality curve decreases hyperbolically in the age interval $\mathrm{A}$ before $t_{c}$ and increases exponentially in the interval $\mathrm{B}$ following $t_{c}$. For those familiar with reliability theory, region A is called the "burn in" phase and B is the "wear out" phase. Using the exponents of the hyperbolic and exponential phases, we introduce a new 2 dimensional map of diseases. This permits the classification of diseases into three broad classes: AS1, AS2 and S. Class AS1 includes all diseases arising from congenital malformations which dominate infant and child mortality; class AS2 includes degenerative diseases such as dementia and Altzheimer's which dominate old age mortality. In class $\mathrm{S}$, which includes most infectious and metabolic diseases, the exponents from both aging phases contribute to positions on the map. Cancer is one of these mixed cases but is closer to class AS2 than AS1. A second line classification is needed to resolve $S$ cases and to this end we introduce a $3 \mathrm{rd}$ dimension, namely (calendar) time. Using historical data we show that in their response to treatment (particularly vaccination), $\mathrm{S}$ diseases fall into three sub-classes. (i) Class E diseases (e.g. measles or meningococcal disease) which have been almost eliminated at all ages (ii) class $\mathrm{C}$ diseases (e.g. tuberculosis) which can be cured but whose cure becomes less effective at old age. (iii) Class $U$ diseases for which radical cures are still unknown. Regarding the future, the fact that the wear-out process of numerous diseases already starts around the age of 25 means that a major extension of the human lifespan beyond 120 certainly also requires to uncover the secret of the "elixir of eternal youth" which has driven timeless human efforts and still seems unlikely in the foreseeable future.

\section{Version of 22 September 2017}

Key-words: death rate, infant mortality, classification, disease, reliability

1: School of Physics, Trinity College Dublin, Ireland. Email: peter_richmond@ymail.com

2: Institute for Theoretical and High Energy Physics (LPTHE), University Pierre and Marie Curie, Paris, France. Email: roehner@1pthe.jussieu.fr 


\section{Introduction}

In the last months of 2016 the question of whether there is a limit to human lifespan has been hotly debated in several physics publications ${ }^{1}$. Here, we examine it in the light of a new classification of diseases.

The medical classification of diseases (also called semiology) is based on clinical symptoms. The classification proposed here is based on completely different data, namely on variables which describe the structure of death rates as a function of age. It will be called a "systems science classification" (for brevity SSC). The term "systems science" refers to the fact that this classification originates from the field of reliability analysis of technical devices.

\section{Link with reliability analysis}

For technical devices the successive phases correspond to the well-known stages of the so-called "bathtub" curve.

(1) Decreasing failure rate: infant stage (also called "burn-in" stage) during which defective items fail and are eliminated.

(2) Constant failure rate: normal service stage characterized by a small number of random failures.

(3) Increasing failure rate: "wear-out" stage during which failures become more and more frequent.

Fig. 1a and 1b suggest that human life comprises similar stages with however the qualification that the constant failure phase covers only a short interval from about 8 to 12 years. For that reason it will be omitted in the subsequent discussion.

The burn-in and wear-out phases are characterized by two symmetrical processes: elimination of defects in the burn-in phase and accumulation of defects in the wearout phase.

\section{Redefinition of infant mortality}

In medical statistics the expression "infant mortality" refers to the first year after birth and during this first year infant death rates (e.g. early, late and post neonatal) are defined with respect to live births and not with respect to the living population at the beginning of the respective time intervals. The $0-1$ year definition has its origin in the recording process of postnatal mortality data. However, it is not a logical definition in the sense that the one-year age interval has no real biological basis. A more objective definition of "infant mortality" is suggested by reliability analysis. For technical devices, infant mortality refers to the entire age interval during which the mortality rate decreases. For humans this corresponds to the period 0-10 years.

\footnotetext{
${ }^{1}$ Richmond et al. 2016b (27 September), Dong et al. 2016 (13 October), Buchanan 2016 (1 November).
} 


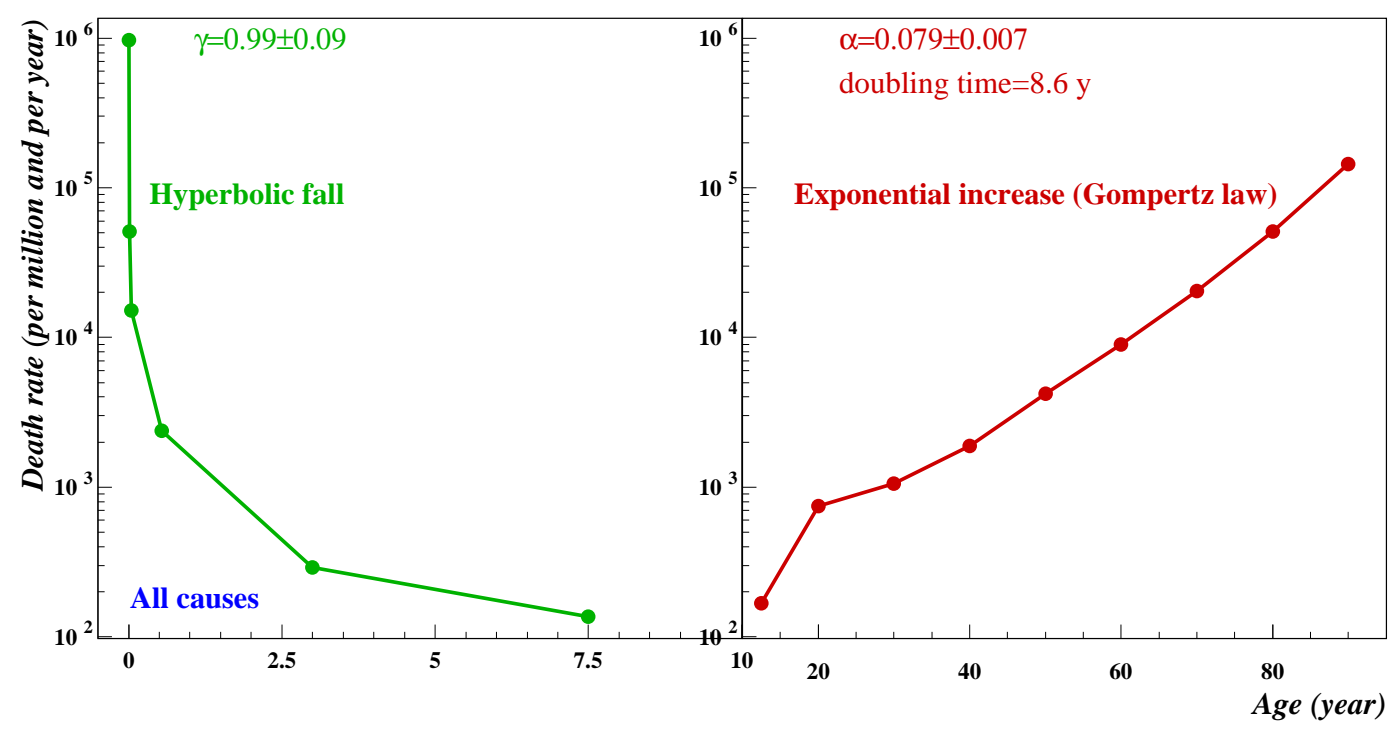

Fig. 1a Death rate from all causes, USA 1999-2014. The $x$ axis represents age from 1 day after birth to 90 years. For 1 day to 10 years the death rate is defined as the standard infant death rate, i.e. $\mu_{b}(t)=$ $|[1 / s(0)](d s / d t)|$ where $t$ is the age, $s(t)$ the number of survivors and $s(0)$ the number of live births. From 10 years to 90 years the death rate is defined as the standard adult death rate (also called force of mortality or hazard rate) i.e. $\mu(t)=|(1 / s)(d s / d t)|$. The $y$-scales are logarithmic. Source: CDC "Wonder" database 1999-2014 (http://wonder.cdc.gov/ucd-icd10.html). All data are average annual death rates over the 16-year long interval 1999-2014.

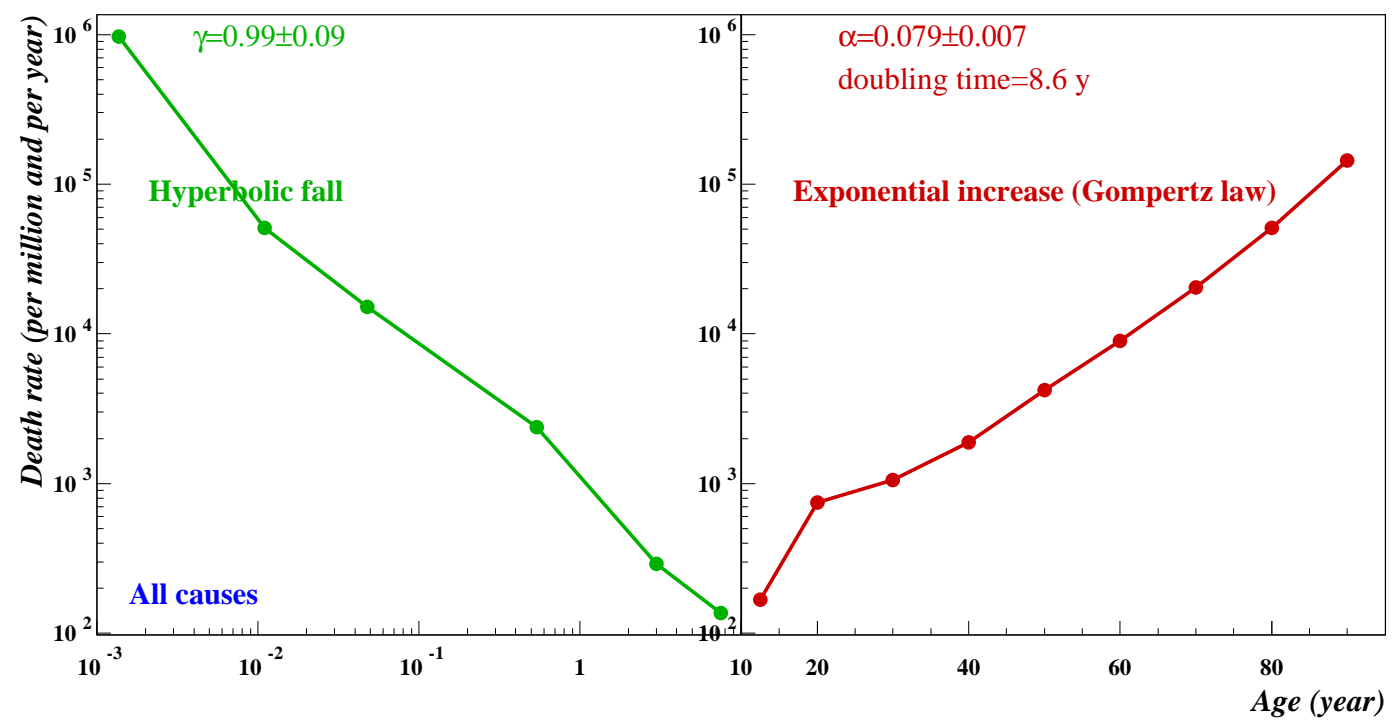

Fig. 1b Death rate from all causes, USA 1999-2014. The only difference with Fig. 1a is that from 1 day to 10 years the $x$ scale is logarithmic. The two straight lines mean that for $(0,10)$ the death rate is hyperbolic of the form $\mu_{b} \sim 1 / t^{\gamma}, \gamma=0.99$ whereas for $(10,90)$ it is exponential in accordance with Gompertz's law $\mu \sim e^{\alpha t}, \alpha=0.079$. It can be noted that due to the huge range of variation of the death rate, using linear scales would produce a completely useless graph in the sense that for $(0,10)$ it would be a vertical line whereas for adult deaths it would be a line superimposed on the $x$ axis. Thus, the $y$ log-scale is not just an option, it is a necessity. Source: Same as in Fig. la. 
It is this definition of infant mortality which will be used throughout this paper.

\section{What is the purpose of the systems science classification?}

Let us say right away that the medical classifications and the SSC have very different objectives.

- The aim of any medical classification is to allow identification of the disease for the purpose of prescribing an appropriate cure. For that reason, the classification must be as detailed as possible. For instance, it is important to distinguish between small cell lung cancer and large cell lung cancer because the treatment is not at all the same.

- On the contrary the SSC provides a broad classification. Its purpose is to uncover patterns and regularities. The practical usefulness of such patterns will be discussed in the conclusion.

\section{Classification of diseases into three broad categories}

Before we define the classification, it should be noted that in principle one needs not only death data but also incidence data. By relying on deaths we actually mix up two phenomena which should be studied separately, that is (i) The emergence of a disease and (ii) How well a disease can be treated.

When a disease can be completely cured it tends to disappear, e.g. smallpox, cholera or measles in developed countries. Fortunately two facts contribute to make death data relevant nevertheless.

- For most diseases there is fairly limited curability for the youngest ( $<1$ day) and oldest ( $>90$ year) age groups. This point is related to the so-called rectangularization $^{2}$ of the death rate curve (see Meslé and Vallin 2002 and also Berrut et al. 2016, p. 403, 412).

- We are mainly interested in relative death levels and the latter are more or less proportional to incidence levels.

\section{Class AS1: diseases in which burn-in predominates}

Fig. 2 shows a typical case in which burn-in plays a predominant role.

Table 1 shows that deaths linked to perinatal problems and congenital malformations dominate death rates in the first month after birth. In subsequent months the share of these two causes decreases. Overall, for the first year they represent $70 \%$ of the deaths, $16 \%$ in the second year and $6.6 \%$ for the nine to ten year age group.

Some congenital malformations may lead to death in the hours after birth whereas others may bring about death only a long time after birth. As a matter of fact, it is

\footnotetext{
${ }^{2}$ In our representation it would rather be a triangularization because in Fig. 1 there is no plateau.
} 


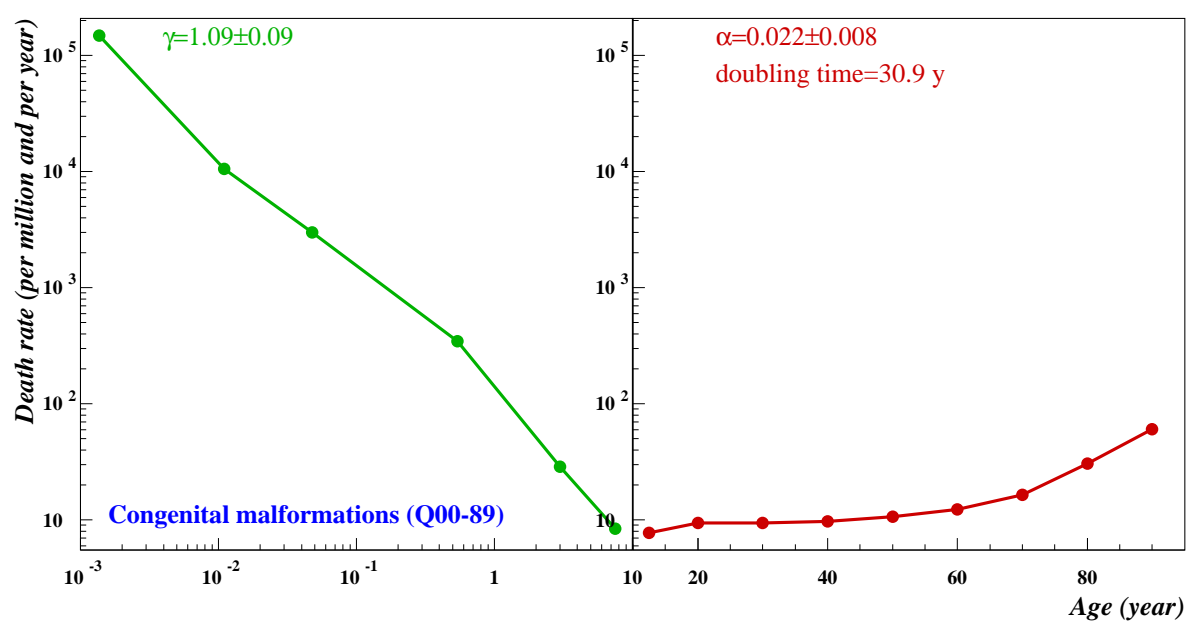

Fig. 2 Death rate due to congenital malformations, USA 1999-2014. On the left, between birth and 10 years, the death rate is divided by $10^{4}$ whereas on the right it is multiplied by a much smaller factor, namely only 7. This shows that there is a very active burn-in process which contrasts with a rather sluggish wear-out process. Sources: Same as in Fig. 1 a

Table 1 Deaths due to anomalies during the infant phase (from birth to 10 years)

\begin{tabular}{lcccccc}
\hline \hline & & & & & & \\
& $0-1^{-}$ & $1^{+}-7^{-}$ & $7^{+}-28^{-}$ & $28^{+}-365^{-}$ & $5 y^{+}-6 y^{-}$ & $9 y^{+}-10 y^{-}$ \\
\hline Delivery problems P00-96 & $79 \%$ & $65 \%$ & $55 \%$ & $7.0 \%$ & $0.62 \%$ & $0.63 \%$ \\
Congenital malform. Q00-99 & $18 \%$ & $26 \%$ & $25 \%$ & $17 \%$ & $7.9 \%$ & $6.0 \%$ \\
P00-96 + Q00-99 & $97 \%$ & $91 \%$ & $80 \%$ & $24 \%$ & $8.5 \%$ & $6.6 \%$ \\
\hline
\end{tabular}

Notes: The percentages are with respect to all deaths in the corresponding age interval. Here $1^{-}$denotes the end of the first day (i.e. $23 \mathrm{~h}$ after birth) whereas $1^{+}$denotes the beginning of the second day (i.e. $25 \mathrm{~h}$ after birth). The last column is for 9 years to 9 years and 365 days. The code numbers following the causes of death are from the ICD-10 classification, that is to say the 10th revision of the "International Classification of Diseases"; in most countries it was introduced between 1995 and 2000. The combined deaths P00-96+Q00-99 decrease sharply in the first year but the fall levels off thereafter.

Source: CDC (Centers for Disease Control): “Wonder" database, 1999-2014.

quite remarkable that even 50 years after birth the cause of death can still be traced back to a congenital malformation.

\section{Class AS2: diseases in which wear-out predominates}

For the case of Alzheimer's disease shown in Fig. 3 there is no $A$ phase in the sense that for this disease the first death occurs in the $10^{+}-15^{-}$age group. A similar shape is observed for other dementia. The connection between such diseases and wear out processes is emphasized in Courchesne et al. (2000).

Fig. 4, adapted from Courchesne et al. (2000), shows that the volume of gray matter (i.e. the external layer of the brain) decreases steadily after the age of 10. It is true that the volume of white matter decreases more slowly but because good functional- 


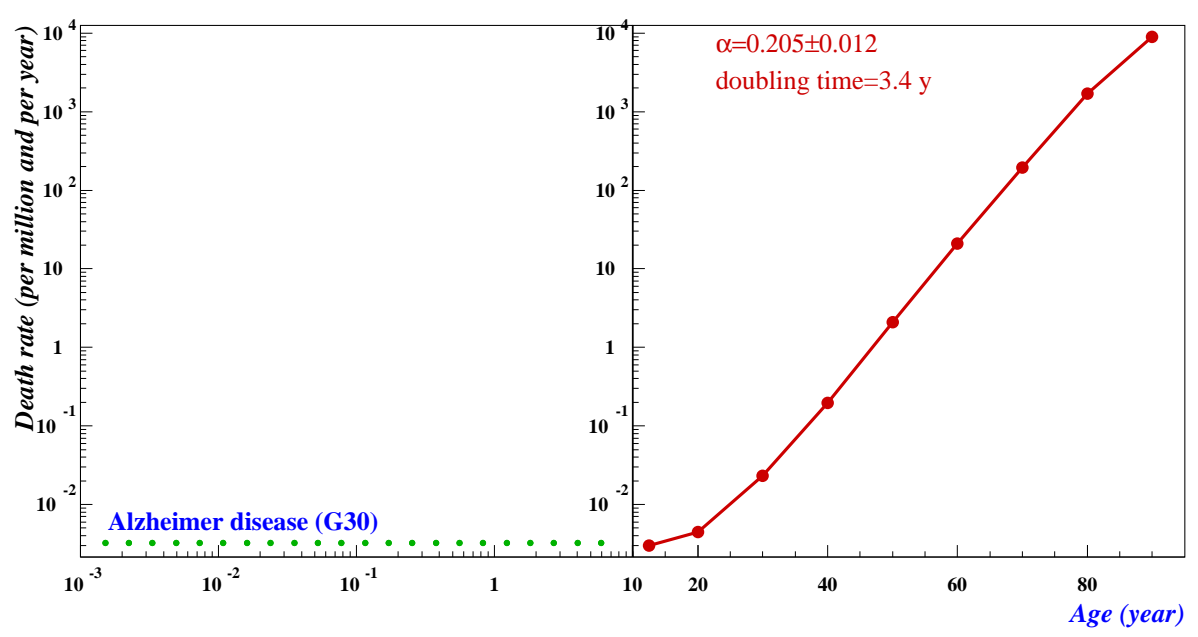

Fig. 3 Death rate due to Alzheimer's disease, USA 1999-2014. The earliest deaths due to Alzheimer's disease are in the $10^{+}-15^{-}$age group; as zero values cannot be shown due to the logarithmic scale on the $y$ axis, the green broken line should be seen as symbolically representing the zero level. Sources: Same as in Fig. 1a

ity requires a combination of both white and gray matter ${ }^{3}$, a loss of capabilities seems inevitable. Moreover, the inset shows that instead of being an "orderly" shrinkage, it is a wear and tear process which distorts the shape of the brain.

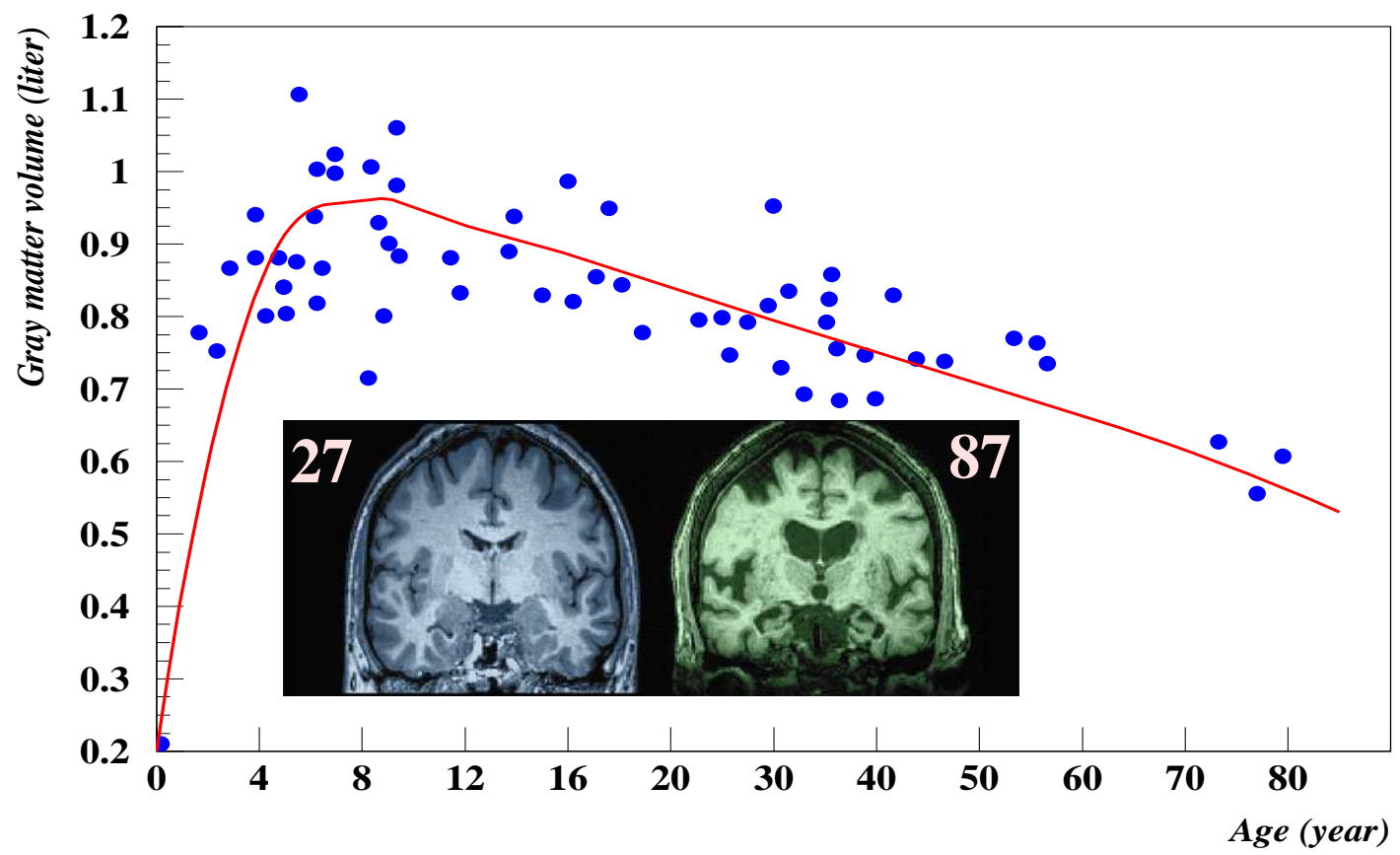

Fig. 4 Decrease of the volume of gray matter. The graph describes quantitatively the shrinkage that the inset shows qualitatively. The data are for 116 healthy volunteers whose mean age was 21.4 years. From the data points it is apparent that the sample comprised only few elderly people; thus, there was nobody between 50 and 65 or over 80. Sources: Adapted from Courchesne et al. (2000) and http://www.discovermagazine.com (inset).

In Fig. 4 the important point is that wear-out starts early. Similarly, bone density

\footnotetext{
${ }^{3}$ One needs only remember that white matter consists of more than 100,000 kilometers of nerve fibers which connect parts of the gray matter with each other.
} 
starts to decrease after the age of 25 , that is to say a long time before osteoporosis becomes a medical problem. As a third example, one can mention human sensitivity to high frequency audio signals which starts to fall after the age of 20, again long before any hearing loss becomes noticeable in common life activities. In short, any attempt to stop (or even slow down) the wear-out process must also start early. In other words, a drug ensuring a long life must by necessity also afford a prolonged youth. It can be noted that in various civilizations and over many past centuries the search for an "elixir of eternal youth" has been a major objective of alchemists.

\section{Intermediate cases}

By "intermediate cases" we mean diseases whose rate decreases substantially in the infant phase and raises significantly in the aging phase.

Common features of diseases due to pathogens

'Bacterial diseases' such as the one shown in Fig. 5a are typically of this kind.

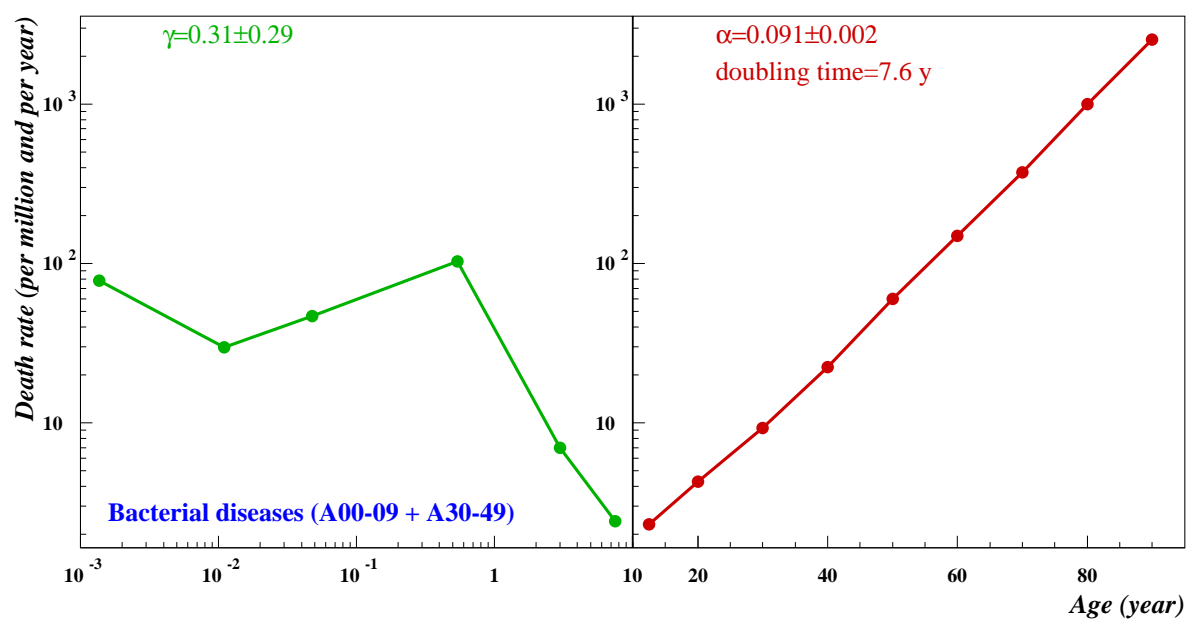

Fig. 5a A case in which there is both burn-in and wear-out: death rate due to bacterial diseases, USA 1999-2014. Based on the ranges of the two graphs one may say that the wear-out process is more considerable than the burn-in process. Sources: Same as in Fig. la

This time, in contrast with Fig. 4, the decline in the infant phase cannot be interpreted as being due to the existence of pre-natal defects. How then should one interpret such a graph? A natural interpretation is to say that the decline is due to a strengthening of the immune system. If correct, this interpretation would lead us to say that there is a weakening of the immune system starting right away after the age of 10 .

Incidentally, we point out here that the peak which occurs between the age of one month and one year is not a statistical fluctuation for it is found in many countries and various time periods. We might speculate that it arises in a similar manner to that observed for fish as they leave the yolk sac and finally forage independently. Thus here perhaps the peak is related to the fact that between 6 and 12 months, babies shift from the anti-bodies given by the mother to their own immune system. 
This interpretation of the dynamics of the immune system can be tested by examining other cases in which one would also expect the immune system to play a role. For instance, in table 2 we examine the case of viral infections,

Table 2 Similarities and differences between diseases due to bacteria or viruses.

\begin{tabular}{llccc}
\hline \hline Disease & $\begin{array}{c}\text { Position of } \\
\text { infant peak } \\
\text { [year] }\end{array}$ & $\begin{array}{c}\text { Exponent of } \\
\text { infant decline }\end{array}$ & $\begin{array}{c}\text { Doubling time of } \\
\text { adult phase } \\
\text { [year] }\end{array}$ \\
\hline Viral infections (A80-B34) & 0.05 & $0.52 \pm 0.21$ & $15.4 \pm 10.5$ \\
Bacterial diseases (A00-09+A30-49) & 0.53 & $0.31 \pm 0.29$ & $7.6 \pm 0.16$ \\
\hline
\end{tabular}

Notes: Although it follows broadly the same pattern, the viral case differs from the bacterial case in two respects (i) the secondary peak occurs earlier (ii) the mortality rate levels off after the age of 50 instead of increasing steadily as in Fig. 5. These features remain unexplained so far. These infant peaks were studied more closely in a separate paper (yet unpublished).

Source: CDC (Centers for Diseases Control): "Wonder" database, 1999-2014.

Difference in adult profiles between bacterial and viral diseases

For viral diseases the increase in the adult phase is much slower than for bacterial diseases. This difference, which can broadly be seen in the last column of Table 2, is confirmed for specific diseases as shown in Fig. 5c,d. Moreover, it appears that for protozoan infections the increase with age is even slower than for viral infections. The reason remains an open question.
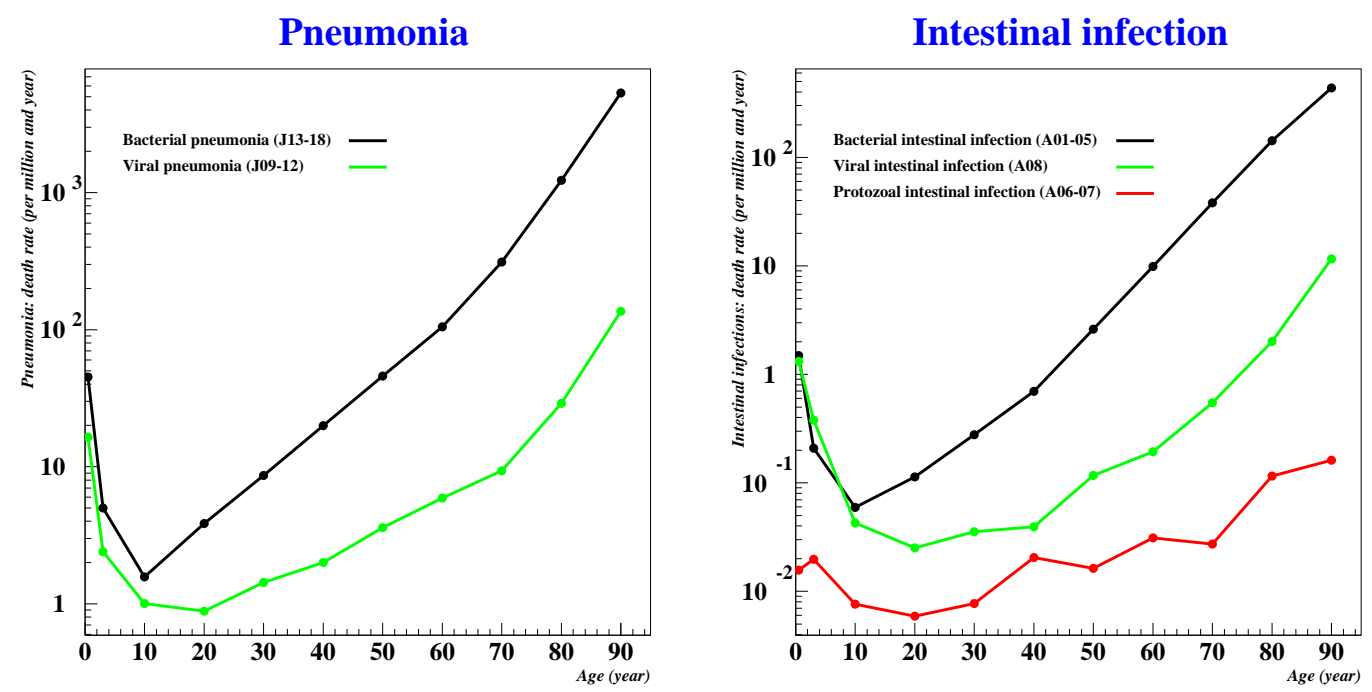

Fig. 5b,c Bacterial, viral and protozoan diseases as a function of age. At the age of 90 the differences in death rates are quite considerable: 40 for bacterial versus viral pneumonia and 1,000 for bacterial versus protozoan intestinal infections. Source: Same as for Fig. 1 a

\section{Distribution of diseases in the $(\gamma, \alpha)$ plane}




\section{Introduction of the $(\gamma, \alpha)$ plane}

The values of the parameters $\gamma, \alpha$ estimated previously for various diseases can now be used to position each disease in the $(\gamma, \alpha)$ plane introduced in Fig. 6.

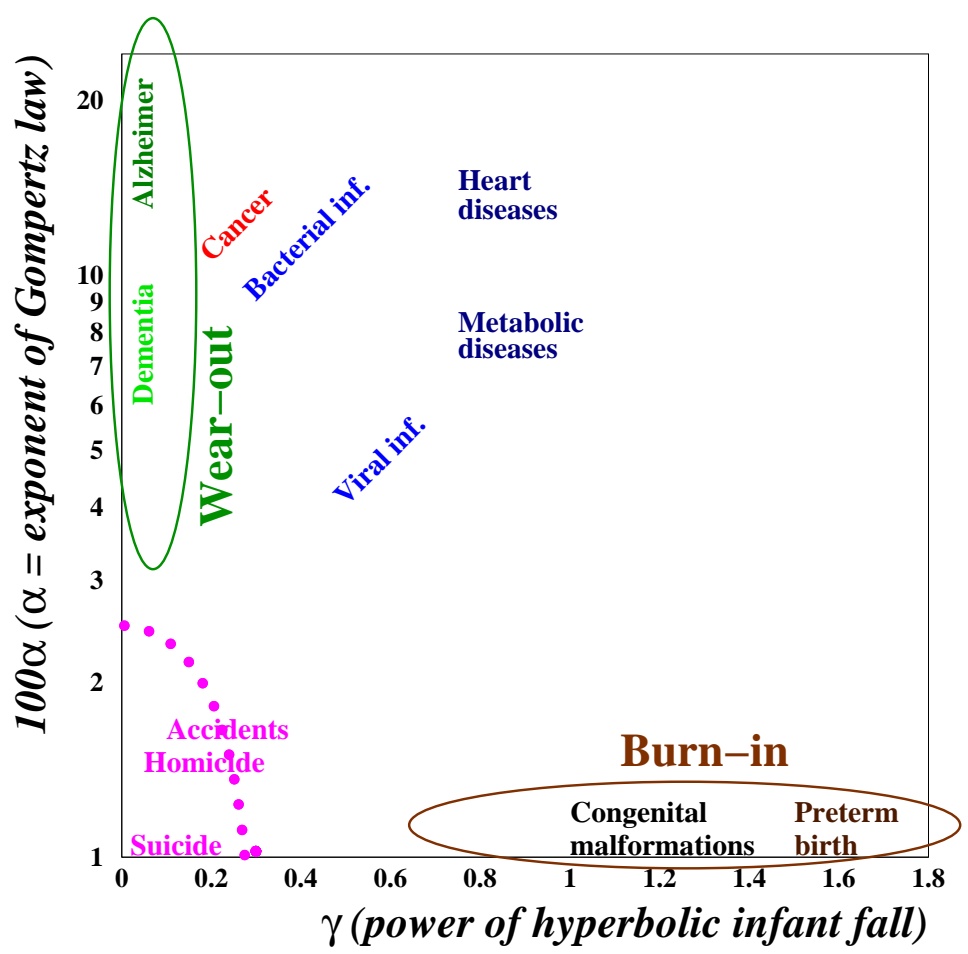

Fig. 6 Location of diseases in the $(\gamma, \alpha)$ plane, USA 1999-2014. $\gamma$ is the power of the hyperbolic fall $\mu_{b} \sim$ $1 / t^{\gamma}$ of the infant phase while $\alpha$ is the exponent of the exponential increase in the adult phase: $\mu \sim e^{\alpha t}$. Along the $\gamma$ axis are all diseases related to congenital defects including those resulting from preterm birth. Along the $\alpha$ axis are all degenerative diseases. In between the remaining diseases may be closer to the first or second group denoting a stronger congenital or degenerative component respectively. The "Metabolic diseases" label refers to diseases due to deficiencies, as for instance diabetes.

\section{What is the status of cancer?}

We now discuss the status of cancer in the present framework.

There is a long standing debate about the status of cancer. Is it a disease that is susceptible of being cured (like tuberculosis, say) or is it a degenerative disease (like Alzheimer's disease, say)? Can our framework give us some clues?

Status of cancer in the present classification. If cancer is due to a few mechanisms that may be knocked out, a global remedy may indeed be conceivable. However, if it is due to a (programmed) weakening of the immune system that hope does not seem realistic. One faces the same problem for technical devices in the sense that it is not always obvious whether an increasing failure rate is due to one isolated hidden defect or to the simultaneous attrition and wear of many components. In the first case the defect may be eliminated, however in the second case one would need to replace almost every part of the device. 


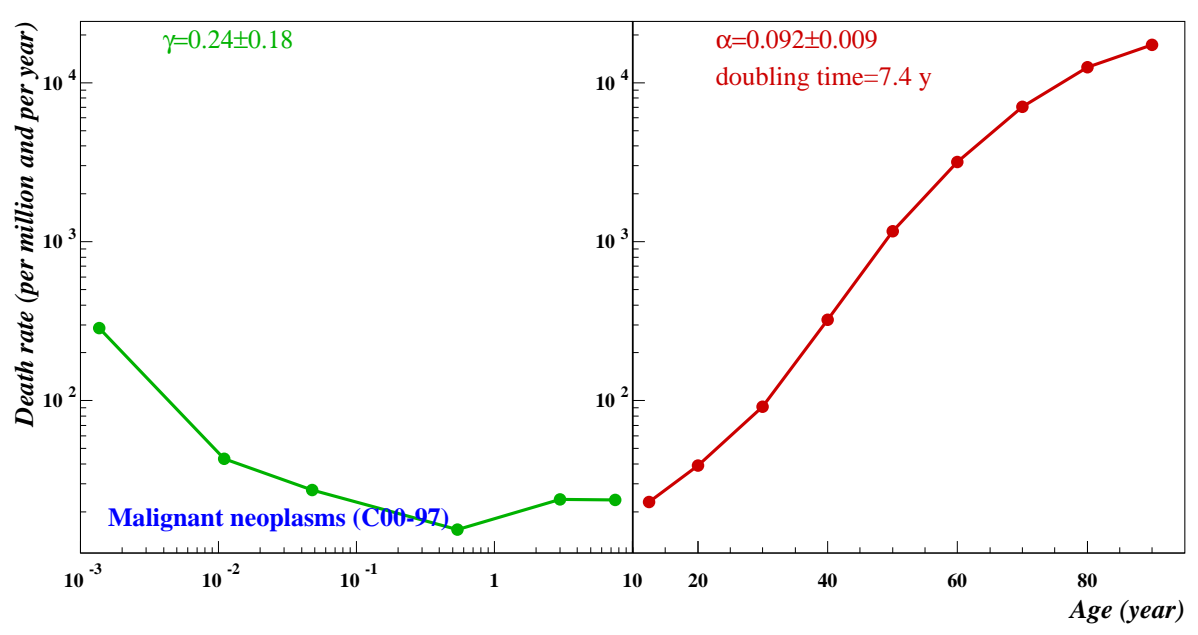

Fig. 7 Cancer death rate, USA 1999-2014. Based on the respective ranges of variation of the infant and adult death rates one may say that the wear-out process is more considerable than the burn-in process. Sources: Same as in Fig. 1a.

Fig. 7 shows that in the infant phase cancer looks very similar to the case of bacterial diseases of Fig. 5 except that there is no secondary peak. In their adult phases the two cases look also very similar both in shape and doubling-time. The case of bacterial diseases was (tentatively) explained by successive changes in the strength of the immune system. Surprisingly for a disease such as tuberculosis for which a treatment is available, its adult phase mortality has a doubling time of about 9.1 years which is not very different from the doubling time of cancer. The same observation holds for other bacterial diseases for which antibiotics are supposed to be highly effective. These features are consistent with a conception in which one assumes that any therapy, no matter how effective, can only help the immune system but cannot replace it. Thus, even for a well-treated disease such as tuberculosis, the therapy barely influences old age death rates (at ages around and above 90-100).

\section{Secondary classification based on response to therapy}

When one follows the historical changes in the course of time of the age-specific death rates for various diseases it becomes quickly obvious that they display different patterns which define a number of distinct classes. In defining these patterns we rely on data for developed countries. Why?

The level of the death rate is conditioned by the number of the hospitals, physicians and more generally by the amount of resources that a society devotes to its health care system. Moreover, in developed countries most vaccines have been introduced several decades ago which means that we can see their effect fairly clearly. Changes in the shape of the death rate may reflect and so to say summarize the complex interaction between a disease and the immune system. In some cases a vaccine may 
completely suppress a disease; that is for instance the case of smallpox or tetanus. On the contrary for tuberculosis the vaccine (as well as other means) was able to reduce considerably the death rate in young and mid-life ages but did not much reduce the rate for elderly people. Thus, this death rate differential markedly modified the shape of the curve. In short, shape changes are likely to reflect meaningful biological differences.

One may distinguish the following classes.

(1) Class E: Marked reduction in death rates together with invariability of the shape. In this case there is simply a vertical downward translation of the curve. The examples shown in Fig. 8a are measles and meningococcal disease. In these cases the death rates were divided by a factor comprised between 5 and 10, a reduction which was as substantial for old age as for mid-life. The most striking case in this class is smallpox which in 1980 has been declared eradicated worldwide by the "World Health Organization". The case of measles is somewhat similar. Although still present in developing countries, it has become so rare in developed countries that its rate is hardly measurable (in the US there were only 19 deaths in 17 years). Leprosy, varicella or tetanus also fall in this class. It can be called the class of eliminated diseases (at least in developed countries).
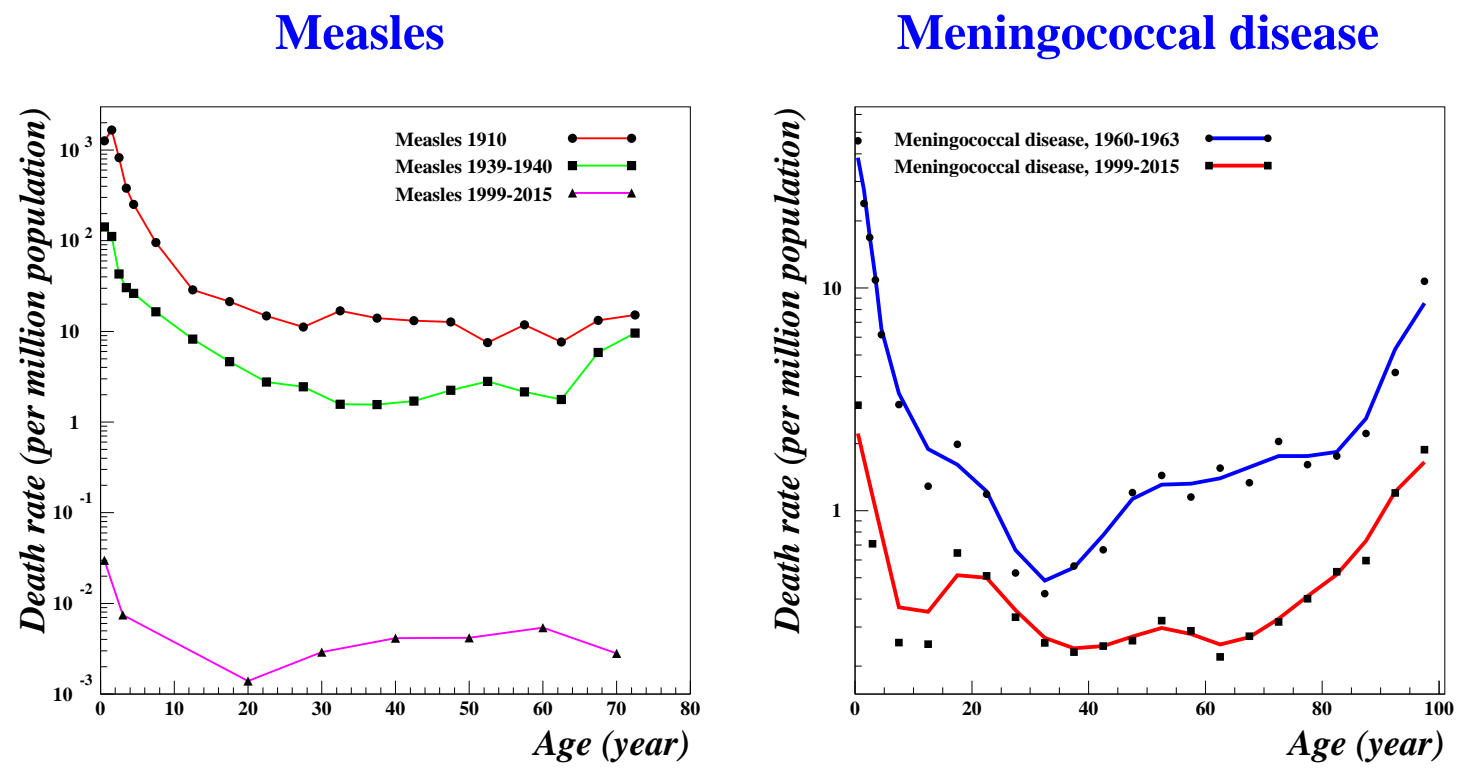

Fig. 8a Class E: measles and meningococcal disease in the United States. The shape of the death rate by age is almost invariant in the course of time but there is a strong reduction in mortality. In contrast with measles which is caused by a virus, the meningococcal disease is caused by a bacterium. Moreover it is not primarily a "childhood disease". The data are for the United States. Because these are diseases with only a small number of deaths they do not follow Gompertz's law. Sources: For 1900, 1940,1960: Mortality Statistics and Vital Statistics for the United Stares. For 1999-2015: Wonder CDC database. 


\section{Tuberculosis}

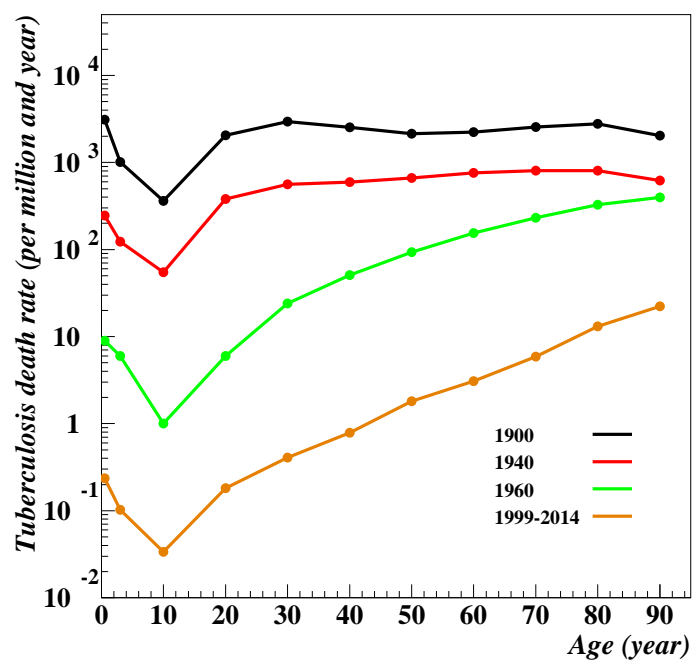

\section{Heart disease}

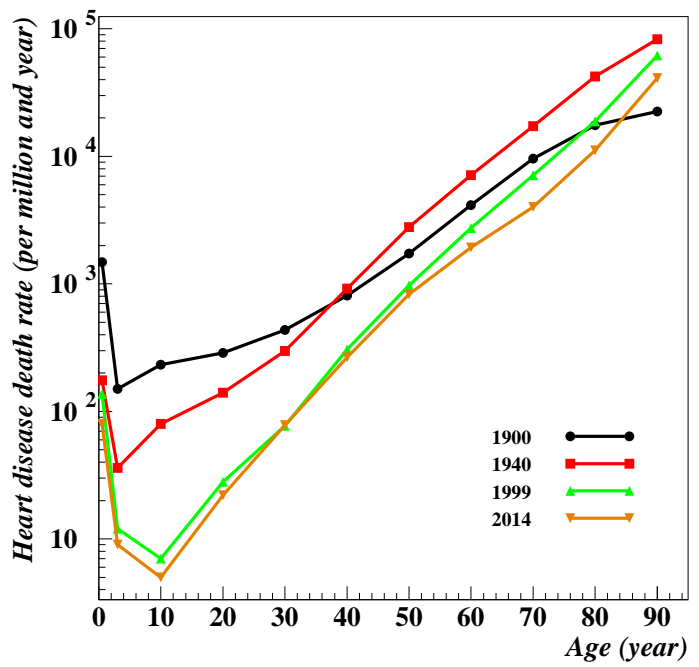

Fig. 8b Class 2: tuberculosis and heart disease in the United States. There is a well-defined pattern in the sense that in the adult phase the slope (that is to say the exponent $\alpha$ ) increases as the global mortality decreases. The fact that for Switzerland almost identical curves are observed suggests that this pattern is shared by other developed countries. The ICD10 code definition of tuberculosis is A16-19. For heart disease it is the combination of the following intervals: I10-15, I20-25, I26-28, I30-51. Sources: Linder and Grove (1947, $p$. 248-254); Grove and Hetzel (1968, p. 378-469); "Wonder" database of the "Center for Diseases Control" $(C D C)$.

\section{Intracerebral accident}

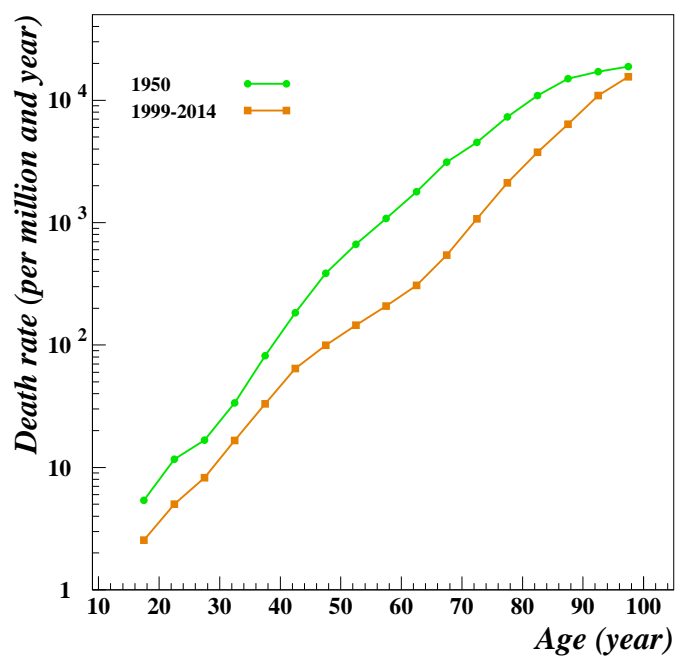

Cancer

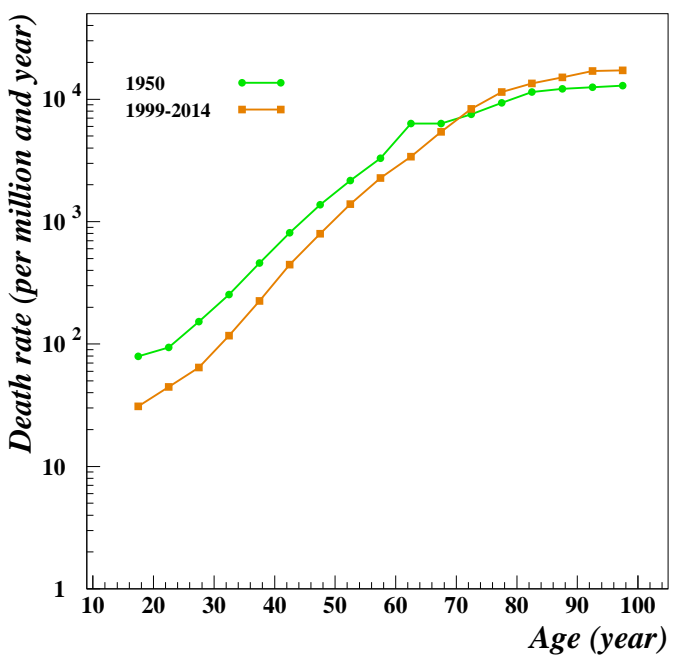

Fig. 8c Class U: intracerebral accident and cancer. in the United States. The ICD10 codes for intracerebral accident and cancer (malignant neoplasms) are I60-64 and C00-97 respectively. Sources: Vital Statistics of the United States 1950, Volume 3; "Wonder" database of the "Center for Diseases Control" (CDC).

(2) Class C: Age-dependent changes resulting in shape modification. This is the case already discussed for tuberculosis. Influenza is very similar. Heart diseases follow the same pattern as shown in Fig. 8b. Epilepsy is a similar case. Because for such diseases the total mortality was considerably reduced it can be said that they are 
cured diseases.

(3) Class U: No major changes. No (or only slight) reduction in death rates and quasi-invariability of the shape. The examples shown in Fig. 8c are intracerebral accidents and cancer.

For the sake of uniformity, the graphs of Fig. 8a,b,c were drawn for US data. However, similar patterns are observed in other developed countries. This was checked particularly for Switzerland, a country whose historical mortality data have been made available on Internet.

Some of the changes described above are fairly easy to interpret. In a general way, all eliminated diseases are infectious diseases. One may be tempted to attribute this elimination to vaccination but in fact the mortality of measles started to fall well before the introduction of the vaccine in 1968. In contrast the diseases in class 2 and 3 are, partially or completely, diseases due to wear-out. For that reason, they are more difficult to cure especially in old age.

Remark The graph for tuberculosis (Fig. 8b) shows that between 1960 and 19992014 the trend has changed in the sense that in this time interval of 40 years the death rate reduction has extended to old age. This is probably related to the introduction in the 1950s of the triple therapy, a combination of three separate medications. Nonetheless, the death rate at the age of 90 remains about 100 times higher than at the age of 10 which means that tuberculosis is still far from belonging to class E.

\section{Conclusion}

The classifications proposed in the paper are summarized in Fig. 9. What can be their usefulness? As already said, there is no claim that it may improve therapy. However, it may give useful indications to decision makers for defining healthcare policy. As an illustration, one can consider the case of cancer.

For cancer the stated objective of many programs is to "beat cancer" or to "win the war on cancer". Here are some typical statements.

- "Those who took part in the overnight relay will not rest until a cure for cancer has been found".

"Twelve hours of fun, friendship and fundraising to beat cancer".

- In his speech after signing the "National Cancer Act" of 1971, President Richard Nixon used the expression "the conquest of cancer", perhaps in a parallel with the conquest of the Moon.

In other words, the set goal is not just to find a cure for specific forms of cancer but to eliminate cancer as a major cause of death, just as tuberculosis was eliminated as a major disease. It can be noted that such an ambitious objective is at odds with the 

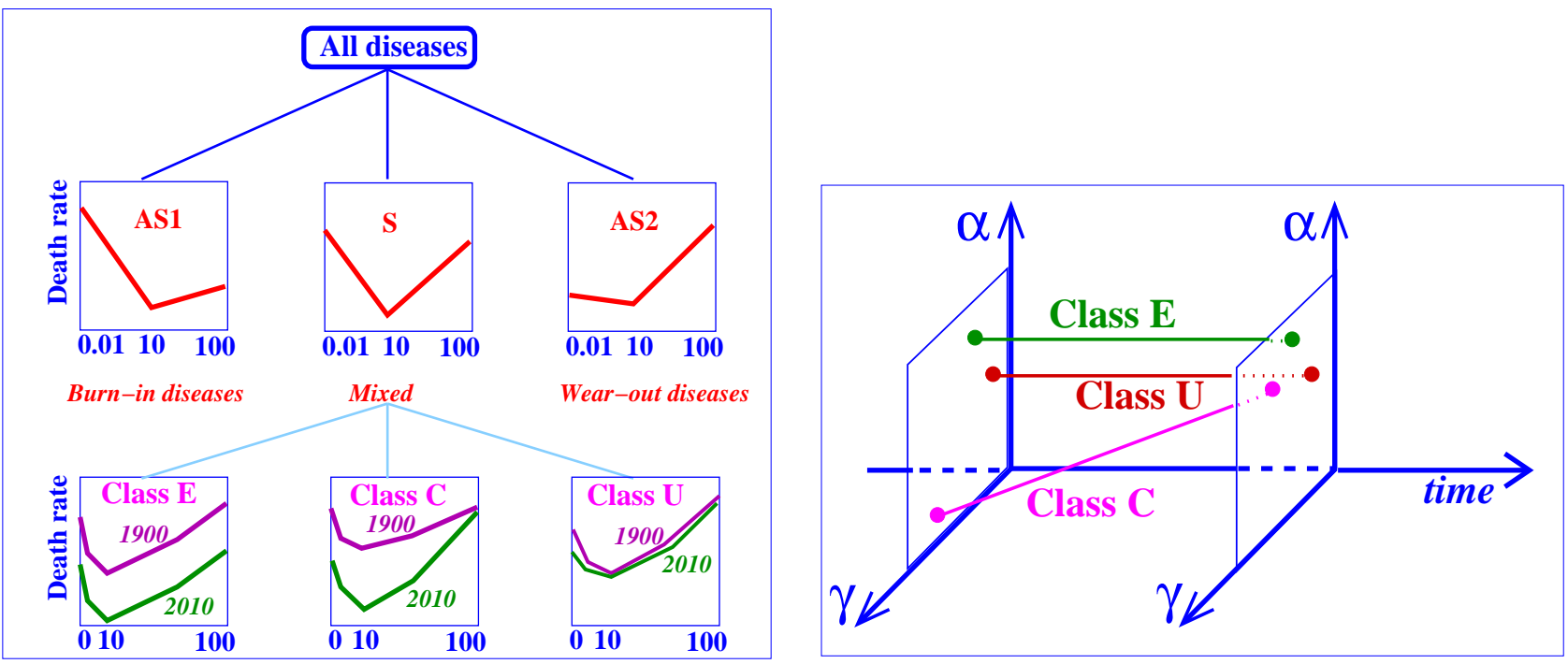

Fig. 9a,b Diagrams summarizing the classifications proposed in the paper. (a) The first-line classification is based on the exponents of the hyperbolic and exponential laws whereas the second-line classification is based on the historical response of diseases to therapy. (b) 3-d representation of shape changes due to response to therapy. Because the difference between class 1 and class 3 involves the reduction in mortality levels, it cannot be seen on this diagram (remember that the parameters $\alpha, \gamma$ describe only the shape).

place of cancer in the classifications proposed in this paper.

Nobody would claim that it is possible to cure completely all forms of heart disease. If one accepts the idea that the heart is a pump the fact that it is limited to a certain number of beats seems reasonable. Yet, for cancer, because its origin is more mysterious, huge amounts of funds are devoted to its elimination. Incidentally, it can be observed that according to an estimate given in Olshansky et al. (1990) elimination of all forms of cancer would increase life expectancy at birth by only 3 years. The reason is that cancer is predominantly a disease of old age which means that its elimination would concern mostly the relatively small group of people who reach such old ages. Moreover, in such age groups there are, apart from cancer, numerous other causes of death.

\section{Appendix A. Data and method}

\section{Data}

In this paper we relied on US statistical data for convenience of access reasons. However, our exploration of the data of other countries (for instance France or Switzerland) convinced us that similar results would be obtained in other industrialized countries.

In this respect, one should mention the the epidemiological data collected and published by the "Global Burden of Disease" group at the initiative of the Lancet journal. 
The complete title is given below under the reference "GBD 2017". Once these data will be organized into an interactive data base they will provide a very convenient and helpful tool.

In the US we used one interactive database, namely "CDC WONDER (1999-2014)", and also the following datasets:

- US mortality data. Until 1937 these annual volumes were entitled "Mortality Statistics"; subsequently their name was changed to: "Vital Statistics of the United States". All these volumes have been digitized and are available on the website of the "Centers for Disease Control". The data are mostly death numbers; rates are given in a few cases only.

- Linder and Grove (1947) plus Grove and Hetzel (1968). These authors compiled the death numbers given in the previous source and computed corresponding rates.

\section{Statistical estimates}

The estimation procedure was facilitated by linearisation of the death rates through a log-log representation for infant death data and a semi-log representation for adult data. Thus, our exponents $\gamma$ and $\alpha$ are linear least squares estimates. In the graphs and in Table 2 the \pm indications are $95 \%$ confidence intervals. Because the data points are very much collinear such confidence intervals are usually fairly small (under 10\%) and do not play any role in our arguments.

The doubling time $T$ was derived from the exponent $\alpha$ through the standard formula: $T=\log 2 / \alpha$.

It can be observed that in contrast to $T$ which is dependent upon the time measurement unit, $\gamma$ is a dimensionless exponent.

\section{References}

Berrut (S.), Pouillard (V.), Richmond (P.), Roehner (B.M.) 2016: Deciphering infant mortality. Physica A 463, 400-426.

Buchanan (M.) 2016: Lifespan limits. Nature Physics 12,989 (November).

CDC WONDER 1999-2014

It is a mortality database set up and maintained by the "Centers for Disease Control". It is available at the following address:

http://wonder.cdc.gov/ucd-icd10.html.

Courchesne (E.), Chisum (H.J.), Townsend (J.), Cowles (A.), Covington (J.), Egaas (B.), Harwood (M.), Hinds (S.),Press (G.A.) 2000: Normal brain development and aging: quantitative analysis at in vivo MR imaging in healthy volunteers. Radiology, volume 216. issue 3.

Dong (X.), Milholland (B.), Vijg (J.) 2016: Evidence for a limit to human lifespan. 
Nature 538,257-259 (13 Oct).

Linder (F.E.), Grove (R.D.) 1947: Vital statistics rates in the United States 19001940. US Government Printing Office, Washington DC.

GBD (Global Burden of Disease) Mortality Collaborators (2016) 2017: Global, regional, and national under-5 mortality, adult mortality, age-specific mortality, and life expectancy, 19702016: a systematic analysis for the Global Burden of Disease Study 2016.

The Lancet Vol.390, No.10100,p.1084-1150, 16 Sep. 2017

[The 360-page appendix of the article gives the names of the 1,500 collaborators and provides worldwide annual death data at national and regional level.]

Grove (R.D.), Hetzel (A.M.) 1968: Vital statistics rates in the United States 19401960. US Government Printing Office, Washington DC.

Meslé (F.), Vallin (J.) 2002: Montée de l'espérance de vie et concentration de l'âge au décès. [Increase in life expectancy and rectangularization of the age-specific death rate.] Institut National d'Etudes Démographiques, Working paper No 108.

Olshansky (S.J.), Carnes (B.A.), Cassel (C.) 1990: In search of Methuselah: estimating the upper limits to human longevity. Science 250, 4981, 634-640.

Olshansky (S.J.), Carnes (B.A.), Désesquelles (A.) 2001: Prospects for human longevity. Science 291, 5508, 1491-1492.

Richmond (P.), Roehner (B.M.) 2016a: Predictive implications of Gompertz's law. Physica A 447,446-454.

Richmond (P.), Roehner (B.M.) 2016b: Is cancer a disease that can be cured? An answer based on a new classification of diseases. arXiv:1609.08285 (http://lanl.arxiv.org/abs/1609.08285) 